\title{
Simposio \\ LA SOCIEDAD CIVIL Y EL ACCESO A LOS MEDICAMENTOS
}

\section{Derechos de propiedad intelectual y acceso a tratamientos: la experiencia brasileña}

\author{
Renata Reis \\ Associação Brasileira Interdisciplinar de AIDS-ABIA, Rio de Janeiro, Brasil
}

La entrada en vigor del acuerdo TRIPS (Trade Related Aspects of Intellectual Property Rights) de la Organización Mundial del Comercio (OMC) estableció patrones mínimos de protección de la propiedad intelectual incluyendo las patentes de productos y procesos farmacéuticos. El argumento dominante era que el fortalecimiento de la propiedad intelectual ayudaría a estimular el desarrollo de las innovaciones, así como el aumento de la transferencia de tecnología entre países, como fue explicitado en la sección que define los objetivos del acuerdo.

En 1996, el año siguiente a la entrada en vigor del acuerdo TRIPS, el tema entró en la agenda de la Asamblea Mundial de la Salud como reflejo de las preocupaciones que la protección de patentes podría tener sobre el acceso a los medicamentos esenciales. Desde entonces, el tema no salió de la agenda de la salud y la evolución del debate se centró inicialmente en el esclarecimiento del derecho de los países a incorporar y hacer uso de las llamadas flexibilidades de protección de la salud pública.

En virtud de la importancia de mantener políticas públicas, como la de acceso universal a tratamientos antirretrovirales y de los desafíos y obstáculos impuestos por las nuevas reglas de protección a la propiedad intelectual, grupos de la sociedad civil brasileña, con el apoyo de organizaciones internacionales, resolvieron aunar esfuerzos para enfrentar el tema, al mismo tiempo relevante y complejo. Así, fue creado en 2001 el Grupo de Trabajo sobre Propiedad Intelectual de la Red Brasileña por la Integración de los Pueblos (GTPI/REBRIP) coordinado por la Asociación Brasileña Interdisciplinar de Sida-ABIA desde su creación. El GTPI está formado por varias organizaciones de la sociedad civil brasileña y por Médicos Sin Fronteras-Brasil, además de algunos investigadores.
Este trabajo trae los aportes más relevantes de este grupo en el escenário nacional del acceso a los tratamientos y los impactos de las normas de propiedad intelectual. EI GTPI actúa principalmente en los siguientes frentes buscando minimizar el evidente impacto negativo del sistema de patentes en Brasil, así:

1. fortalecimiento de la cooperación sur-sur para el cambio de experiencias en el sujeto y posibles acciones conjuntas entre la sociedad civil;

2. acciones de propugnación (advocacy) y proposición de acciones jurídicas y administrativas, en el intento de disminuir el impacto de las regulaciones de propiedad intelectual en el acceso a los medicamentos esenciales y en la salud pública en general;

3. formación y movilización de la opinión pública en relación con el impacto social de los acuerdos comerciales en propiedad intelectual, $y$

4. seguimiento de los foros internacionales que discutan el tema de la propiedad intelectual y el acceso a los medicamentos.

Las estrategias que vienen siendo propuestas por la sociedad civil brasileña revelan los importantes desafíos en el presente y para el futuro en los países en desarrollo, y pueden ser vistos desde tres ángulos: (a) perspectiva de producto por producto, (b) el sistema nacional de patentes, y (c) la necesidad de la reforma del sistema internacional de patentes.

El primero está relacionado con el constante seguimiento de nuevos medicamentos que están siendo adoptados por el consenso terapéutico, así como de las barreras para el acceso a ellos. Se cree que es muy importante el fortalecimiento de la cooperación entre los países en desarrollo, ya que probablemente enfrentarán los mismos problemas para los mismos medicamentos. 
El segundo está relacionado con el sistema nacional como un todo y cómo estimular el desarrollo de medicamentos para enfermedades olvidadas. Esta perspectiva más amplia representa desafíos estructurales para la implementación constante de las políticas de salud.

Finalmente, reflexionar sobre una fuente alternativa para estimular el desarrollo de nuevos medicamentos sin pasar necesariamente por la apropiación intelectual, especialmente el sistema de patentes, que viene demostrando su carácter impeditivo al acceso de las poblaciones más vulnerables y tampoco han demostrado ser eficaces en el caso de tecnologías que no son atractivas al mercado.

\section{Referencias}

1. Angell M. A verdade sobre os laboratórios farmacêuticos. Rio de Janeiro: Record; 2008.

2. Bermudez JAZ, Oliveira MA, Chaves G C. O Acordo TRIPS da OMC e os desafios para a saúde pública. En: Bermudez JAZ, Oliveira MA, Esher A, org. Acceso a medicamentos: derecho fundamental, papel del Estado. Rio de Janeiro: ENSP/FIOCRUZ; 2004. p. 69- 89,

3. Reis R, Vieira M, Chaves G. Acesso a medicamentos e propriedade intelectual no Brasil: reflexões e estratégias da sociedade civil. En: Direitos de propriedade intelectual e acesso aos antirretrovirais: resistência da sociedade civil no sul global. Rio de Janeiro: Associação Brasileira Interdisciplinar de AIDS; 2011.

4. 't Hoen E. 2008. The global politics of pharmaceutical monopoly power - drug patents, access, innovation and the application of the WTO Doha Declaration on TRIPS and public health. Disponible en: http:// www.msfaccess.org/fileadmin/user_upload/ medinnov_accesspatents/01- 05_BOOK_tHoen_ PoliticsofPharmaPower_defnet.pdf.

5. World Health Organization, 2006. Report of the Commission on Intellectual Property Rights, Innovation and Public Health. Available at: http://www. who.int/intellectualproperty/documents/thereport/ ENPublicHealthReport.pdf.

\title{
La familia de las artemisias ofrece una politerapia poderosa contra las enfermedades tropicales y la disentería
}

Pierre Lutgen

\author{
IFBV-BELHERB, Hostert, Luxemburgo
}

Como expertos en el campo de la certificación ambiental y de salud (ISO 14000 y OHSAS 18000) nos hemos enfrentado en nuestras misiones internacionales con la tremenda carga de las enfermedades tropicales y la disentería. Si es verdad que las malas prácticas ambientales tienen un impacto sobre la salud y las enfermedades, lo contrario es todavía más cierto: las enfermedades y la pobreza tienen un efecto desastroso sobre el ambiente, la polución de las aguas, la deforestación, el inadecuado uso de la energía y los deslizamientos de tierra.

Las enfermedades caen dentro de dos grupos distinguibles, los bien financiados y los mal financiados, como la leishmaniasis, la malaria, el cólera, el dengue, la enfermedad de Chagas, la filariasis, la dracunculiasis, la amebiasis, la fascioliasis, el tracoma, la hidrofobia, la úlcera de Buluri, la giardiasis, etc. Los fondos que se adjudican actualmente para combatir las enfermedades son notoriamente bajos comparados con los costos de atención de la salud para enfermedades más comunes. Estas enfermedades desatendidas son una gran causa de muerte, incapacidad, perturbación social y económica para millones de personas. La productividad perdida, las oportunidades de educación perdidas y el alto costo de la salud causados por las enfermedades contagiosas tienen un impacto importante en las familias y en las comunidades.

Cada año mueren 9,5 millones de personas debido a enfermedades infecciosas, casi todas viven en los países en desarrollo. Los niños son particularmente vulnerables a las enfermedades infecciosas. La neumonía, la diarrea y la malaria son las causas principales de muerte ente los niños menores de 5 años; la malaria cerebral puede causar un deterioro mental permanente.

De manera conjunta con asociaciones en una docena de países y universidades en África y Suramérica, estamos concentrando nuestros esfuerzos en la lucha contra la malaria y la disentería.

La malaria, al contrario de muchas creencias, ha sido también una enfermedad común en países templados, aun en Siberia. Después de la segunda guerra mundial se llevó a cabo un gran esfuerzo para erradicar esta enfermedad, con éxitos notables en casi todos los países del norte: Sicilia, España, Florida, Cuba, Rusia, Ontario, Grecia. 
La herramienta ideal para esto fue el DDT, pero los abusos en su empleo llevaron a que las organizaciones ecologistas se preocuparan y el producto fue prohibido antes de que se consiguiera la erradicación total en los países pobres del sur. Una sobrerreacción con consecuencias notorias porque, a pesar de cientos de estudios médicos que intentaron demostrar una eventual toxicidad humana del DDT, hasta hoy no se ha conseguido documentar ningún efecto negativo en la salud humana y, en septiembre de 2006, la OMS levantó la prohibición del uso de DDT para el rociado interno residual. De hecho, el DDT actúa sobre los mosquitos más por su efecto repulsivo que por su toxicidad. El rociado interno residual es una luz de esperanza para millones de niños que mueren de malaria.

Aunque le damos la bienvenida al uso del DDT para rociado interno residual que puede salvar de inmediato miles de vidas, creemos que el futuro está en los productos herbáceos más que en los insecticidas químicos. El extracto de neem (Asadirachta indica), por ejemplo, tiene fuertes propiedades repulsivas e insecticidas.

Pero, más allá de las estrategias preventivas basadas en mosquiteros para camas e insecticidas, las terapias basadas en medicina herbácea pueden ser muy efectivas y ganarán importancia. El 70\% de la población mundial todavía se asienta en ellas. Una planta juega un rol clave en este enfrentamiento novedoso: Artemisia annua. Durante los últimos años hemos acumulado evidencia científica que muestra que si un té de esta planta se toma durante siete días (50 g en 20 tazas) la infección de la malaria se cura por completo, con un mínimo riesgo de recaída ().

Si se toma durante más de 7 días puede reducir la gametocitemia y la transmisión del hombre al mosquito. Los chinos han conocido a esta planta por más de 2.000 años y en ninguna parte se ha notado ninguna señal de resistencia a la terapia con esta planta. Sin embargo, las píldoras para la terapia combinada de artemisia han dado durante el año pasado señales alarmantes de resistencia en una docena de países. Probablemente, porque carecen de los efectos sinérgicos de los polifenoles y de los aceites esenciales presentes en la hierba. Un análisis extenso $\left(^{2}\right)$ de tisanas Artemisia annua de varios países muestra que la hierba de Luxemburgo es más rica en aceites esenciales (1.8 cineol, limoneno, terpineol, etc.) y escopoletina. En todos los ensayos in vitro e in vivo muestra propiedades antipalúdicas equivalentes o mejores, aunque sea más pobre en artemisinina que las otras. Parece que otras variedades de artemisia, como Artemisia afra $\left({ }^{3}\right)$ utilizada en la Sudáfrica o Artemisia apiacea utilizada en la China, también tengan buenos efectos terapéuticos contra Plasmodium falciparum, Es por eso que hablamos de la familia de las artemisias en el titulo de la ponencia.

El efecto más inesperado fue descubierto en Kenia ( $\left.{ }^{4}\right)$ : la tisana de $A$. annua es profiláctica. En las escuelas en donde los alumnos cultivan la hierba y consumen su copa diaria de tisana, el ausentismo cayó de $30 \%$ a $0 \%$ porque ya no sufren de paludismo. $\mathrm{Y}$, de hecho, en estudios hechos en Luxemburgo hemos descubierto que la $A$. annua conduce a una notable activación de los linfocitos.

Para poder coordinar mejor nuestros esfuerzos, hemos lanzado este año a Belherb (Asociación para la Promoción de la Medicina Herbal). Se basa en el trabajo de una docena de profesores universitarios y médicos de Bélgica y Luxemburgo. Las medicinas herbales de China e India han hecho milagros durante siglos. Con las modernas herramientas espectroscópicas queremos conocer mejor su cinética farmacológica.

En nuestrotrabajo de investigación en Luxemburgo hemos descubierto que $A$. annua tiene un fuerte efecto esterilizador $\left(^{5}\right)$ sobre el agua contaminada. De hecho, una taza de té añadida a un litro de agua de río provee agua perfectamente potable. Este efecto fue confirmado por varias universidades europeas y, también, por universidades en Senegal y África Central, y en Colombia por Nestor Aguirre de la Universidad de Antioquia (6).

La Universidad de Antioquia en Colombia (PECET) confirmó que $A$. annua tiene buenas propiedades terapéuticas para la leishmaniasis y la fasciola hepática.

La Universidad de Belgrado $\left({ }^{7}\right)$ confirmó que el té de $A$. annua tiene citotoxicidad contra líneas de células malignas humanas: el adenocarcinoma HeLa del cuello uterino, el melanoma maligno Fem-x y BG, la leucemia mieloide K562, el adenocarcinoma de mama MDA-MB-361 y el cáncer de colon LS174.

En el campo de la malaria, los resultados más alentadores fueron obtenidos por nuestros asociados africanos en las universidades de Dakar, Bangangte, Bangui y Yaoundé. Sus resultados están disponibles por pedido.

Hemos iniciado plantaciones en una docena de países de África y Suramérica, y hemos alcanzado 
niveles de producción para la comercialización de productos fitofarmacéuticos en varios de estos países. Pero el objetivo final de nuestra ONG es que esta hierba esté presente en todas las huertas tropicales.

Los científicos de Belherb están muy concientes de que se necesita trabajo adicional de investigación para confirmar y extender los descubrimientos mencionados en cooperación con nuestros asociados académicos en el sur. En caso de que esto fuere confirmado, sería revolucionario. Cada día mueren 20.000 niños de malaria, cólera, diarrea, leishmaniasis, etc.

\section{Referencias}

1. Chougouo RD. Comparative study of the quality and efficiency of Artemisia annua grown in Cameroun. MIM conference, Kenya, Oct 2009.
2. Hansen G, Chougouo RD. Rapport de Stage, Institut Supérieur des Sciences de la Santé, Cameroun, et Laboratoire National de Santé, Luxembourg, 15 septembre 2010.

3. Liu NQ, et al. Journal of Ethnopharmacology. 2010;108:121-7.

4. Arudot T. Kenyatta University, Nairobi, comunicación personal.

5. Lutgen P. Congrès Maladies Tropicales, Luxembourg, 6-7 avril 2009.

6. Aguirre N. Grupo GAIA de la Universidad de Antioquia. Interacción SODIS y artemisia para la obtención de agua potable epilimética de la ciénaga de Ayapel, Córdoba, Colombia, octubre de 2009.

7. Juranić Z, Lutgen P, Matić I. Institute of Oncology and Radiology of Serbia, Belgrade, Serbia. EACR conference, Stocholm, July 2010.

\title{
La sociedad civil en el debate global sobre innovación, propiedad intelectual y salud pública
}

\author{
Francisco Rossi \\ Fundación IFARMA, Bogotá, D.C., Colombia
}

\section{El debate global}

Aunque para muchos la protección a la propiedad intelectual es el mejor mecanismo para promover la innovación farmacéutica, este escrito parte del entendido de que existe una crisis global en el modelo de innovación farmacéutica. Crisis que tiene varias expresiones, de las cuales cabe destacar:

- Estancamiento de la innovación expresada en una reducción progresiva del número de nuevas moléculas a pesar del crecimiento en el ámbito de la protección a la propiedad intelectual (patentes, protección de datos, patentes para usos, pequeñas modificaciones, combinaciones) y del creciente número de países asumiendo compromisos en materia de propiedad intelectual para medicamentos.

- Baja calidad de la innovación desde una perspectiva sanitaria con una proliferación progresiva de innovaciones tipo me too, de patentes "perennes" (en inglés, evergreening).

- Innovación dirigida por el mercado; las inversiones se concentran en productos de alta rentabilidad, que suelen preferentemente dirigirse a los problemas de salud de países desarrollados, abandonando los problemas de salud de los pobres y de los países pobres. Lo que se conoce internacionalmente como neglected diseases.

- Mayor gasto en mercadeo (marketing) que en investigación y desarrollo. Se configura, entonces, un modelo de innovación enormemente costoso, que responde mejor a las prioridades de rentabilidad de la industria que a las necesidades de salud.

- Concentración en unas pocas empresas a nivel global en detrimento de empresas y grupos de investigación de los países en desarrollo.

El resultado más importante de este modelo de innovación en crisis, es que los gobiernos del mundo observan que el gasto en medicamentos crece con una rapidez mayor que los ingresos de los sistemas de salud y que la economía en su conjunto. El año anterior el Parlamento Europeo organizó un foro con el título "¿Puede Europa financiar el modelo de innovación farmacéutica?". La respuesta fue bastante categórica; es imperativo encontrar esquemas alternos al modelo actual porque los recursos ya no son suficientes en varios países, y más temprano que tarde, no serán suficientes en ninguno de ellos. En el caso de Colombia, conviene recordar que la Emergencia Social y la Reforma de la Ley 1438 de 2010, estuvieron motivadas en el crecimiento incontrolable del gasto en medicamentos no POS, concentrado en los medicamentos más nuevos y más costosos, muchos de ellos en condición de exclusividad.

El debate se ha concentrado en si podrían existir otros modelos de innovación diferentes a la protección de la propiedad intelectual. Y sí que los hay. En general, se ha propuesto desde una 
perspectiva sociológica y económica, identificar opciones que no liguen el estímulo a la innovación con el precio final del producto. Que privilegien el esfuerzo de la innovación sobre el marketing.

Hay muchas alternativas. Algunas diseñadas específicamente para tornar atractivas las inversiones en enfermedades olvidas o desatendidas, como las alianzas público-privadas, cuyo mejor ejemplo internacional es la DNDi (Drugs for Neglected Diseases Iniciative), otras para reducir o compartir los riesgos, como los contratos de compra anticipada, utilizados especialmente para el desarrollo de vacunas, y otras para separar por completo la innovación del precio como es el caso de las diferentes modalidades de premios, fondos de premios y la propuesta de un fondo global dirigido a financiar los éxitos en la innovación, para las enfermedades elegidas como prioritarias.

Este debate tuvo un espacio privilegiado durante los dos años de discusiones del grupo intergubernamental de trabajo de la OMS que desembocó en la Resolución de la Asamblea Mundial de la Salud № 61.21.

El papel de los gobiernos y de las Naciones Unidas

La definición de las políticas públicas de salud en general y de innovación en medicamentos en particular, no ha sido fácil, en especial en los países en desarrollo y hemos de decir que aún queda mucho camino por recorrer.

Esa tarea, que por naturaleza atañe a los gobiernos, se ha quedado corta. Baste traer a la memoria los porcentajes del PIB que dedicamos los países en desarrollo a la promoción de la innovación, y compararlos con lo que se destina en países de la OECD. En un mundo en el que los costos de la innovación farmacéutica tienden a crecer, es fácil saber en manos de quiénes terminarán quedando las innovaciones y sus réditos.

Preocupa sí una tendencia, creciente en nuestros gobiernos y en nuestras universidades para importar el modelo que hoy los países ricos se preguntan si van a poder sostener. Me refiero al mito de estimular las patentes y la cultura de la confidencialidad de datos en los grupos de investigación, y la tendencia a introducir el esquema de relaciones universidadindustria basado en propiedad intelectual y las diferentes modalidades de transferencia de la misma, que ya están tan cuestionadas en otras latitudes, pero que aquí apenas empiezan.

Las organizaciones de las Naciones Unidas que en su origen buscaban evitar una tercera guerra mundial, se han convertido en los espacios de debate, de análisis, de desarrollo de programas y estrategias para conseguir sociedades más justas, más equitativas, más igualitarias, más democráticas. Pero puesto que son organizaciones de los gobiernos, y los gobiernos parecen tener una creciente tendencia a responder a los grupos de poder, las Naciones Unidas también se han quedado cortas.

\section{El papel de la sociedad civil}

Decir que se quedan cortos los gobiernos y las Naciones Unidas es una manera elegante de reconocer que en el mundo de hoy suceden demasiadas cosas inaceptables, cualquiera que sea la perspectiva política que uno quiera asumir.

Un tercio de la población del mundo tiene hambre. En pocos años enfrentaremos una escasez de agua que, probablemente, afecte a más de mil millones de personas, mientras hoy estamos ante una trágica epidemia de inundaciones en un planeta que pareciera haber perdido sus buenas costumbres y sus buenas rutinas estacionales.

Un tercio de la población mundial, según cálculos de la organización Mundial de la Salud, no tiene acceso a medicamentos para aliviar el dolor, curar las enfermedades y evitar las secuelas.

Alguien tiene que hacer algo y es por eso que existen las organizaciones no gubernamentales (ONG). Los gobiernos y las Naciones Unidas gastan demasiado tiempo, esfuerzos y dinero, en mirarse a sí mismos y en responder a quienes los sostienen.

Desde una perspectiva histórica, han sido organizaciones de la sociedad civil las que han conseguido poner freno a la escalada de precios y gasto, y a la protección creciente a la propiedad intelectual. Fueron las ONG las que forzaron a las 39 multinacionales a retirar su demanda contra la ley de medicamentos de Sudáfrica. Fue la sociedad civil del Brasil la que se abanderó de la declaración de interés público para algunos antirretrovirales, y fue la sociedad civil en Tailandia la que rodeó al gobierno para la expedición de licencias obligatorias. Y fue la Declaración de Rio de Janeiro la que puso los fundamentos del texto final de la Resolución 61.21 de la Asamblea Mundial de la Salud, mayoritariamente preparada por las ONG.

Nombres como Médicos sin Fronteras, Health Action International, Knowledge Ecology International, Acción Internacional para la Salud, organizaciones de diversas iglesias, Treatment Action Campaign, Oxfam, entre otros, se hicieron familiares para todos los involucrados en estos debates.

Una evaluación de las patentes en Colombia Ifarma, en asocio con CIDEPRO, el centro para el desarrollo de productos de la Universidad de 
Antioquia, realizó un análisis de las patentes farmacéuticas concedidas en Colombia entre 2004 y 2008 para evaluar hasta dónde han contribuido a estimular la innovación en general, la innovación local y la transferencia de tecnología.

Se identificaron 439 patentes concedidas en el período. Prácticamente todas a empresas extranjeras multinacionales. Estados Unidos, Suiza, Alemania y Francia son los países más frecuentes. Entre los fabricantes se destacan F. Hoffmann-La Roche, con el $11 \%$, seguido por Pfizer y Boehringer Ingelheim con el $7 \%$ y $6 \%$, respectivamente. Colombia en el periodo de estudio, cuenta con solo dos patentes sobre excipientes, un porcentaje irrelevante. Hay una proporción importante (35 $\%$ ) de patentes tipo Markush, que cubren un gran número de moléculas que podrían limitar la investigación local.

Colombia ha sido considerada como un país exigente, en el que solamente se conceden patentes a innovaciones genuinas que cumplen a cabalidad los requisitos técnicos y legales. Sin embargo, en el análisis realizado se observa un relajamiento en la aplicación de los criterios de patentes.

De las 439 patentes otorgadas, cerca del $60 \%$ ya no están vigentes, por no pagar las tasas de mantenimiento. Dado el bajo costo de las tasas, se plantean dos hipótesis que pudieran explicar estos vencimientos: la exclusividad se puede conseguir por otros mecanismos más eficaces (marcas, tecnología, regulación), o que pudiera existir un interés de bloqueo a las líneas de investigación local. 